Contemporary Mathematics

\title{
A Tropical Version of the Schauder Fixed Point Theorem
}

\author{
G.B. Spiz and G.L. Litvinov \\ Abstract. A tropical version of the Schauder fixed point theorem \\ for compact subsets of tropical linear spaces is proved.
}

\section{Introduction}

It is well-known that every continuous mapping from a compact subset of a locally convex space to this subset has a fixed point (the Schauder fixed point theorem [1]). There exists a correspondence (and analogy) between important, interesting, and useful constructions and results of the traditional mathematics over fields and analogous constructions and results over idempotent semirings and semifields, i.e., semirings and semifields with idempotent addition (the idempotent correspondence principle, see [2, 3, 4]). In the framework of this analogy a tropical/idempotent version of the Schauder fixed point theorem is proved (see Theorem 2 below).

Note that topologies in tropical/idempotent analysis do not coincide with standard topologies in the traditional functional analysis and collections of compact subsets of spaces of functions do not coincide too. We shall examine applications of our results in a separate paper. In particular, our results could be used to prove that homogeneous (but nonlinear in general) operations in topological idempotent linear spaces have eigenvectors. This is closely related to asymptotic behaviour of

2000 Mathematics Subject Classification. Primary: 46T99, 16Y60, 06A99; Secondary: 06A11, 06F07.

Key words and phrases. Tropical analysis, idempotent analysis, Schauder fixed point theorem.

This work is supported by the RFBR grant 08-01-00601 and the joint RFBR/CNRS grant 05-01-02807.

(C) 0000 (copyright holder) 
infinite extremals in dynamic optimization problems with infinite planning horizon, see [5]- 7]. For example, it is possible to generalize the results of [5, 6] to the case of discontinuous utility functions and kernels of Bellman operators.

\section{Basic definitions and notations}

In the present paper, we shall use some ideas and terminology from [8]-[10]. We recall that an idempotent semigroup (IS) is defined to be an additive semigroup equipped with commutative addition $\oplus$ so that the relation $x \oplus x=x$ holds for every element $x$. Any IS can be treated as a set ordered by the standard (partial) order: $x \preceq y$ if and only if $x \oplus y=y$. It is easily seen that this order relation is well defined and $x \oplus y=\sup \{x, y\}$. For an arbitrary subset $X$ of an idempotent semigroup, we set $\oplus X=\sup (X)$ and $\wedge X=\inf (X)$ under the condition that the corresponding right-hand sides exist. An idempotent semiring (ISR) is defined to be an IS equipped with associative multiplication $\odot$ with unity (denoted by $\mathbf{1}$ ) such that both of the distributivity relations are satisfied. An idempotent semigroup $V$ equipped with multiplication $\odot$ by elements from an idempotent semiring $K$ such that the relations $a \odot(b \odot x)=(a \odot b) \odot x,(a \oplus b) \odot x=a \odot x \oplus b \odot x, a \odot(x \oplus y)=a \odot x \oplus a \odot y$, and $\mathbf{0} \odot x=x \odot \mathbf{0}=\mathbf{0}$ hold for any $a, b \in K$ and $x, y \in V$ is called an idempotent semimodule over the idempotent semiring $K$.

The set $\mathbb{R}$ of all real numbers is a commutative ISR with respect to operations $\oplus=\max$ and $\odot=+$. Denote this semiring by $\mathbb{R}_{\oplus}$; we shall equip $\mathbb{R}_{\oplus}$ with the standard topology of $\mathbb{R}$, so we shall treat $\mathbb{R}_{\oplus}$ as a topological space. Note that $\mathbb{R}_{\oplus}$ has no zero element $\mathbf{0}$ (as a semiring); if we adjoin this element then we obtain the well-known max-plus algebra $\mathbb{R}_{\max }=\mathbb{R}_{\oplus} \cup\{\mathbf{0}\}=\mathbb{R}_{\oplus} \cup\{-\infty\}$ or tropical algebra. Of course, $\mathbb{R}_{\oplus}$ and $\mathbb{R}_{\max }$ have the unity element $\mathbf{1}=0$ and the standard order in $\mathbb{R}_{\oplus}$ coincides with the usual one.

An idempotent semimodule over $\mathbb{R}_{\oplus}$ is called an idempotent $\mathbb{R}_{\oplus^{-}}$ space, or $\mathbb{R}_{\oplus}$-space. The semiring $\mathbb{R}_{\oplus}$ is an idempotent $\mathbb{R}_{\oplus}$-space over itself. A homomorphism from a $\mathbb{R}_{\oplus}$-space $V$ to $\mathbb{R}_{\oplus}$ is called a linear functional on $V$. For arbitrary set $T$ denote by $B(T)$ the $\mathbb{R}_{\oplus}$-space of all bounded mappings from $T$ to $\mathbb{R}_{\oplus}$ equipped with the corresponding pointwise operations.

Let $V$ be an arbitrary partially ordered set (e.g., $V$ is an idempotent semimodule with respect to its standard order), $a, b \in V$. We shall use 
the following notations for intervals and half-intervals:

$$
\begin{aligned}
& {[a, b]=\{x \in V \mid a \preceq x \preceq b\},} \\
& (\cdot, a]=\{x \in V \mid x \preceq a\}, \\
& {[a, \cdot)=\{x \in V \mid a \preceq x\} .}
\end{aligned}
$$

Suppose that $X$ is a subset of an idempotent $\mathbb{R}_{\oplus}$-space $V$ and $X \supset$ $[a, b]$ for arbitrary $a \in X$ and $b \in X$. Then we shall say that $X$ is $o$ convex, see [11]. We shall say that a topology on $V$ is locally o-convex if every element $x \in V$ has a basis of o-convex neighborhoods. Suppose that $V$ is equipped with an o-convex topology such that for arbitrary $v \in V$ the mapping $r \mapsto r \odot v$ (from $\mathbb{R}_{\oplus}$ to $V$ ) is continuous and the half-intervals $(\cdot, v]$ and $[v, \cdot)$ are closed. Then we shall say that $V$ is a topological $\mathbb{R}_{\oplus}$-space.

Denote by $V^{*}$ the set of all continuous linear (over $\mathbb{R}_{\oplus}$ ) functionals on $V$. The set $V^{*}$ is an $\mathbb{R}_{\oplus}$-space with respect to the corresponding pointwise operations. We shall say that $V$ is regular, if for every $x, y \in$ $V, x \neq y$, there exists a functional $w \in V^{*}$ such that $w(x) \neq w(y)$. The topology generated by the basis of all sets of the form $\{x \in V \mid$ $a<w(x)<b\}$ for $a, b \in \mathbb{R}_{\oplus}, w \in V^{*}$ will be called a $\oplus$-weak topology.

Suppose that $V$ and $W$ are topological $\mathbb{R}_{\oplus}$-spaces and $f$ is a mapping from $V$ to $W$. This mapping is called $\oplus$-weakly continuous if for every $w \in W^{*}$ the mapping $w f: V \rightarrow \mathbb{R}_{\oplus}$ is continuous.

Suppose that $V$ is an $\mathbb{R}_{\oplus}$-space and $x, y \in V$. We shall write $x \gg y$ if there exists an element $r>\mathbf{1}\left(r \in \mathbb{R}_{\oplus}\right)$ such that $r \odot y \preceq x$. Define subsets $\mathcal{D}_{x}(r) \subset V$ by the following formula:

$$
\begin{aligned}
\mathcal{D}_{x}(r) & =\left\{y \in V \mid r \odot x \gg y \gg r^{-1} \odot x\right\} \\
& =\{y \in V \mid r \odot x \gg y \text { and } r \odot y \gg x\} .
\end{aligned}
$$

The topology generated by the basis of all sets of the form $\mathcal{D}_{x}(r)$ for $r>1$ will be called uniform. The uniform topology is metrizable. The corresponding metric can be defined, e.g., by the formula:

$$
d(x, y)=\arctan \left(\inf \left\{r \in \mathbb{R}_{\oplus} \mid r^{-1} \odot x \preceq y \preceq r \odot x\right\}\right) .
$$

In the space $B(X)$ of all bounded real functions defined on a set $X \neq \emptyset$ the uniform topology is defined by the metric

$$
d(f, g)=\sup _{x \in X}|f(x)-g(x)| .
$$

\section{Topological $\mathbb{R}_{\oplus}$-spaces}

Lemma 1. Let $V$ be an arbitrary $\mathbb{R}_{\oplus}$-space. Then the sets of the form $\mathcal{D}_{v}(l)$ for $l>\mathbf{1}$ form a basis of neighborhoods of the point $v \in V$ with respect to the uniform topology. 
PROOF. It is necessary to check that for every $y \in \mathcal{D}_{x}(l)$ there exists $r>1$ such that $\mathcal{D}_{y}(r) \subset \mathcal{D}_{x}(l)$. We have $l \odot x \gg y$ and $l \odot y \gg x$, so there exists $p>\mathbf{1}$ such that $l \odot x \succeq p \odot y$ and $l \odot y \succeq p \odot x$. We claim that any $r$ such that $\mathbf{1}<r<p$ is good enough. For any $z \in \mathcal{D}_{y}(r)$, we have $z \gg r^{-1} \odot y$ and $r \odot y \gg z$. It follows that $l \odot z \gg l \odot r^{-1} \odot y \gg p^{-1} \odot l \odot y \succeq x$, and on the other hand $l \odot x \succeq p \odot y \gg r \odot y \gg z$. Thus $z \in \mathcal{D}_{x}(l)$. So $\mathcal{D}_{y}(r) \subset \mathcal{D}_{x}(l)$ because $z \in \mathcal{D}_{y}(r)$ is an arbitrary element.

Proposition 1. Let $V$ and $W$ be $\mathbb{R}_{\oplus}$-spaces. Suppose that $f: V \rightarrow W$ is a nondecreasing mapping such that $f(r \odot v) \preceq r \odot f(v)$ for all $r \succeq \mathbf{1}$, $v \in V$. Then the mapping $f$ is continuous with respect to the uniform topology.

Proof. From Lemma 1 it follows that it is sufficient to prove that the preimage of $\mathcal{D}_{f(x)}(l)$ contains a neighborhood of $x$. In fact the preimage of $\mathcal{D}_{f(x)}(l)$ contains $\mathcal{D}_{x}(l)$. Indeed, suppose that $y \in \mathcal{D}_{x}(l)$. Then $l \odot x \succeq r \odot y$ and $l \odot y \succeq r \odot x$ for some $r \in(\mathbf{1}, l)$. So we have $l \odot r^{-1} \odot f(x) \succeq f\left(l \odot r^{-1} \odot x\right) \succeq f(y)$ and $l \odot r^{-1} \odot f(y) \succeq f\left(l \odot r^{-1} \odot y\right) \succeq$ $f(x)$. Therefore, we have $l \odot f(x) \gg f(y)$ and $l \odot f(y) \gg f(x)$, that is $f(y) \in \mathcal{D}_{f(x)}(l)$ and the proposition is proved.

Corollary 1. For every $\mathbb{R}_{\oplus}$-space, both addition $\oplus$ and multiplication by a number are continuous with respect to the uniform topology.

Proposition 2. For every topological $\mathbb{R}_{\oplus}$-space $V$ the following statements hold:

(1) The topology of $V$ is majorized by the uniform topology of $V$, i.e. every open subset of $V$ is open for the uniform topology.

(2) The space $V$ is a topological $\mathbb{R}_{\oplus}$-space with respect to the uniform topology.

ProOF.

(1) Suppose that $x \in V$ and $U$ is a neighborhood of $x$. Let us show that $U$ contains a neighborhood of $x$ with respect to the uniform topology. The space $V$ is locally o-convex, so we can assume that $U$ is an o-convex set. The mapping $r \mapsto r \odot x$ is a continuous mapping from $\mathbb{R}_{\oplus}$ to $V$, so $U$ contains $r \odot x$ and $r^{-1} \odot x$ for some $r>\mathbf{1}$. Thus $\mathcal{D}_{x}(r) \subset U$ and the statement is proved.

(2) It is obvious, that the uniform topology is o-locally convex and mapping $r \mapsto r \odot x$ is continuous. From the statement (1) it follows that all the half-intervals of the form $(\cdot, a]$ and $[a, \cdot)$ are closed. Thus the proposition is proved. 
The proof of the following proposition is straightforward.

Proposition 3. If $T$ is a finite nonempty set, the uniform topology for $B(T)$ is the topology of pointwise convergence, that is the usual topology of the Euclidean space.

Below we shall equip $B(T)$ with the uniform topology.

Proposition 4. Suppose that $V$ is a regular topological $\mathbb{R}_{\oplus}$-space. Then $V$ is a topological $\mathbb{R}_{\oplus}$-space with respect to its $\oplus$-weak topology.

ProOF. It is easy to see that the $\oplus$-weak topology is o-convex and the mapping $r \mapsto r \odot a$ is continuous under this topology. Let us show that the sets of the form $(\cdot, a]$ and $[a, \cdot)$ are closed. Suppose that $x$ is an element of the closure of $(\cdot, a]$ relative to the $\oplus$-weak topology. Then $w(x) \preceq w(a)$ for every functional $w \in V^{*}$, so $w(a \oplus x)=w(a)$. Hence $a \oplus x=a$ because $V$ is regular, so $x \preceq a$, that is $x \in(\cdot, a]$ and the set $(\cdot, a]$ is closed. For the set $[a, \cdot)$ the proof is similar. So the proposition is proved.

\section{Convex subsets in topological $\mathbb{R}_{\oplus}$-spaces}

Let $V$ be an idempotent semimodule over an idempotent semiring $K, X$ a subset of $V$, and $p: X \rightarrow K$ a function such that $\oplus p(x)=\mathbf{1}$. The element $\bigoplus_{x \in X}(p(x) \odot x)$ is called a $\oplus$-convex combination of all the elements $x \in X$. A subset $X \subset V$ is called $\oplus$-convex if $X$ contains every $\oplus$-convex combination of elements of every finite subsets of $X$, see [12]-15] for general definitions and constructions of this type. We shall say that a subset $X \subset V$ is a-convex if there exists the $\oplus$-convex combination $\bigoplus_{x \in X}(p(x) \odot x) \in X$ for each function $p: X \rightarrow K$ such that $\oplus p(X)=\mathbf{1}$.

From these definitions it follows that every $\oplus$-convex set is a subsemigroup with respect to the idempotent addition $\oplus$ and every $a$ convex set is bounded with respect to the standard order (see Section 2 above). Of course, every $a$-convex set is $\oplus$-convex.

Proposition 5. Let $V$ be a topological $\mathbb{R}_{\oplus}$-space, $X$ its compact subsemigroup. Then there exists $\oplus X \in X$.

Proof. For $v \in X$ we set $X(v)=\{x \in X \mid v \preceq x\}=[v, \cdot) \cap X$. For each finite subset $A \subset X$ we have $\oplus A \in \bigcap_{v \in A} X(v)$, so the collection $\{X(v)\}$ is a centered family of closed subsets of $X$. The set $X$ is 
compact, so there exists an element $x \in \bigcap_{v} X(v)$. By our construction $x \succeq X$ and $x \in X$, so $x=\oplus X$. Thus there exists $\oplus X$ and $\oplus X \in X$. The proposition is proved.

Corollary 2. Under the conditions of Proposition 5 for arbitrary subset $Y$ of $X$ there exists the sum $\oplus Y$ and this sum is an element of the intersection of all closed subsubsemigroups containing $Y$. Moreover, for each $w \in V^{*}$ we have $w(\oplus Y)=\oplus w(Y)$.

Proof. Denote by $\widehat{Y}$ the intersection of all closed subsemigroups containing $Y$; the set $\widehat{Y}$ is a compact semigroup, so it is possible to apply Proposition 5 and the first statement of the corollary is proved. To prove the second statement it is sufficient to prove that $\oplus Y=\oplus(\widehat{Y})$. But it follows from the obvious fact: the set $\{x \in V \mid x \preceq b\}$ is a closed subsemigroup for each $b \in V$.

Proposition 6. Let $V$ be a topological $\mathbb{R}_{\oplus}$-space. Each compact $\oplus$ convex subset $X$ of $V$ is a-convex.

Proof. From Proposition 5 it follows that for each subset $Y$ of $X$ the sum $\oplus Y$ exists and $\oplus Y \in X$. Let $p: X \rightarrow K$ is a function such that $\oplus p(X)=\mathbf{1}$. Denote by $\widehat{p}$ the convex combination $\bigoplus_{x \in X}(p(x) \odot x)$. If $p(v)=\mathbf{1}$ for an element $v \in X$, then $\widehat{p}=\bigoplus_{x \in X}(p(x) \odot x \oplus v)$. For each $x \in X$ the element $p(x) \odot x \oplus v$ belongs to $X$, hence $\widehat{p} \in X$. If the function $p$ does not reach its maximum, then for an arbitrary number $r<\mathbf{1}$ we set $p_{r}(x)=r^{-1} \odot(p(x) \wedge r)$ and $\widehat{p_{r}}=\bigoplus_{x \in X}\left(p_{r}(x) \odot x\right)$; recall that $p(x) \wedge r=\inf \{p(x), r\}=\min \{p(x), r\}$. By construction we have $\oplus p_{r}(X)=\bigoplus_{x \in X} p_{r}(x)=\mathbf{1}$ and $p_{r}(v)=\mathbf{1}$ for an element $v \in X$. By construction we have $\widehat{p} \preceq \widehat{p}_{r} \preceq r^{-1} \odot \widehat{p}$, so $\widehat{p}_{r}$ converges to $\widehat{p}$ with respect to the uniform topology as $r$ tends to 1 . From Proposition 2 it follows that $\widehat{p}_{r}$ converges to $\widehat{p}$ in $V$ as $r$ tends to $\mathbf{1}$, so $\widehat{p} \in X$ because $X$ is compact. The proposition is proved.

Let $V$ be a topological $\mathbb{R}_{\oplus}$-space, $X$ its subset. We set

$$
\operatorname{env}(X)=\left\{y \in V \mid\left(\exists x \in X, r \in \mathbb{R}_{\oplus}\right) r \odot x \preceq y\right\} .
$$

It is clear that $\mathbb{R}_{\oplus} \odot X \subset \operatorname{env}(X)$.

Proposition 7. Let $V$ be a topological $\mathbb{R}_{\oplus}$-space, $X$ its a-convex subset. Then there exists a mapping $\pi: \operatorname{env}(X) \rightarrow X$ such that $\pi$ is continuous with respect to the uniform topology, $\pi(x)=x$, and $\pi(r \odot x) \in \mathbb{R}_{\oplus} \odot x$ for all $x \in X, r \preceq \mathbf{1}$. 
Proof. Set

$$
M=\{(x, y) \in X \times \operatorname{env}(X) \mid(\exists r \preceq \mathbf{1}) r \odot x \preceq y\} .
$$

For $(x, y) \in M$ we set

$$
\begin{aligned}
r_{x}(y) & =\sup \{r \preceq \mathbf{1} \mid r \odot x \preceq y\}, \\
m(y) & =\bigoplus_{(z, y) \in M} r_{z}(y), \\
n_{x}(y) & =m(y)^{-1} \odot r_{x}(y), \\
p(y) & =\bigoplus_{(z, y) \in M} r_{z}(y) \odot z, \\
\pi(y) & =\bigoplus_{(z, y) \in M} n_{z}(y) \odot z=m(y)^{-1} \odot p(y) .
\end{aligned}
$$

By construction we have $m(y) \preceq \mathbf{1}$ and $\bigoplus_{x \in X} n_{x}(y)=\mathbf{1}$ for $y \in$ $\operatorname{env}(X)$. Since $\pi(y)$ is a $\oplus$-convex combination of elements of $X$ the element $\pi(y)$ belongs to $X$. We have $m(y) \odot n_{x}(y) \odot x=r_{x}(y) \odot x \preceq y$; hence $m(y) \odot \pi(y) \preceq y$. By construction we have $m(x)=r_{x}(x)=$ $n_{x}(x)=\mathbf{1}$ for $x \in X$, so $x=n_{x}(x) \odot x \preceq \pi(x) \preceq x$. Thus $\pi(x)=x$ and $\pi$ is a retraction $\operatorname{env}(X) \rightarrow X$.

Let us prove that $\pi$ is continuous. Since $\pi(x)=m(x)^{-1} \odot p(x)$ and the multiplication by coefficients is a continuous mapping $\mathbb{R}_{\oplus} \times V \rightarrow V$ with respect to the uniform topology it is sufficient to show that $m$ and $p$ are continuous for the uniform topology. This statement follows from Proposition 1. Thus Proposition 7 is proved.

Note that similar constructions were used in [12, 14].

Proposition 8. Suppose that $T$ is a finite nonempty set, $X$ is a compact $\oplus$-convex subset of $B(T)$ and $Y$ is the traditional (usual) convex hull of $X$ in the Euclidean space $B(T)$. Then there exists a continuous mapping $\pi: Y \rightarrow X$ such that $\pi(X)=X$.

Proof. From Proposition 6 it follows that the set $X$ is $a$-convex. It is clear that $Y \subset \operatorname{env}(X)=B(T)$, so it is possible to apply Proposition 7 .

The following theorem is a tropical/idempotent version of the Brauer fixed point theorem.

Theorem 1. Suppose that $T$ is a nonempty finite set, $X$ is a compact $\oplus$-convex subset of $B(T)$, and $f$ is a continuous mapping from $X$ to $X$. Then $f$ has a fixed point. 
Proof. Suppose that $Y$ is the traditional convex hull of $X$ in the Euclidean space $B(T)$ and $\pi: Y \rightarrow X$ is the continuous mapping (retraction) discussed in Proposition 7. Then $Y$ is a compact convex subset (in traditional sense) in the Euclidean space $B(T)$. From the Brauer fixed point theorem it follows that the mapping $f \pi$ has a fixed point $x \in Y$. In fact $x \in X$ because $f \pi(Y) \subset X$. Hence $\pi(x)=x$ and $x$ is a fixed point for the mapping $f$. Theorem 1 is proved.

\section{Main results}

Suppose that $V$ is a topological $\mathbb{R}_{\oplus}$-space, $X$ is a compact $\oplus$-convex subset of $V, T$ is a nonempty finite subset of $V^{*}$. Consider the mapping

$$
i: V \rightarrow B(T)
$$

defined by the formula $i(v): v \mapsto t(v)$, where $v \in V$ and $t \in T$. Obviously, the mapping $i$ is linear and continuous.

Lemma 2. There exists a continuous mapping $p: B(T) \rightarrow X$ such that $i(p(f))=f$ for each $f \in i(X)$.

Proof. For $f \in B(T)$ we set $p(f)=\bigoplus_{x \in X, i(x) \preceq f} x$. By Proposition 6, $X$ is $a$-convex. Hence $p(f) \in X$, and for $r \succeq \mathbf{1}$ and $f \in B(T)$ we have $r^{-1} p(r \odot f) \oplus p(f) \in X$. We have $i(p(f)) \preceq f$. Using this inequality and the linearity of $i$, we obtain $i\left(r^{-1} p(r \odot f) \oplus p(f)\right) \preceq r^{-1} \odot r \odot f \oplus f=f$. So we have $r^{-1} p(r \odot f) \preceq p(f)$, that is $p(r \odot f) \preceq r \odot p(f)$. By construction the mapping $p$ is nondecreasing; so from Proposition 1 it follows that $p$ is continuous for the uniform topology on $V$. From Proposition 2 it follows that $p$ is continuous with respect to the initial topology (on $V$ ) which is weaker than the uniform topology. Thus Lemma 2 is proved.

Proposition 9. Suppose that $V$ is a topological $\mathbb{R}_{\oplus}$-space, $X$ is its compact $\oplus$-convex subset, $f$ is a $\oplus$-weakly continuous mapping from $X$ to $X$, and $T$ is a nonempty finite subset of $V^{*}$. Then there exists an element $x \in X$ such that $w(x)=w(f(x))$ for each $w \in T$.

Proof. Suppose that $i: V \rightarrow B(T)$ is the mapping defined in the beginning of this section (before Lemma 2) and $U=i(X)$. As $i$ is linear and continuous, $U$ is a compact $\oplus$-convex subset of $B(T)$. From Lemma 2 it follows that there exists a continuous mapping $p: U \rightarrow X$ such that $i(p(w))=w$ for every functional $w \in U$. The formula $g(w)=$ $i(f(p(w)))$, where $w \in U$, generates a mapping $g: U \rightarrow U$. This mapping is continuous, $U$ is a compact $\oplus$-convex subset of $B(T)$. So, by Theorem 1 , the mapping $g$ has a fixed point $u \in U$. Set $x=p(u)$. Since 
$i(f(p(u)))=u$, we have $i(x)=i(p(u))=u=i(f(p(u)))=i(f(x))$, that is $w(x)=w(f(x))$ for each $w \in T$. The proposition is proved.

The following theorem is a tropical/idempotent version of the Schauder fixed point theorem.

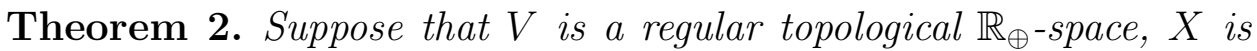
its compact $\oplus$-convex subset, and $f$ is a $\oplus$-weakly continuous mapping from $X$ to $X$. Then $f$ has a fixed point $x \in X$.

Proof. For every nonempty finite subset $T$ of $V^{*}$ we define a set $S(T)$ by the formula

$$
S(T)=\{x \in X \mid w(x)=w(f(x)) \text { for each } w \in T\} .
$$

By construction this set is closed; by Proposition 9 it is nonempty. Obviously, $S\left(T_{1}\right) \cap S\left(T_{2}\right)=S\left(T_{1} \cup T_{2}\right)$; so the family of all sets of the form $S(T)$ is a centered family of closed subsets of the compact set $X$. Hence this family has a nonempty intersection. Let $x$ be an element of this intersection. By construction we have $w(x)=w(f(x))$ for all $w \in V^{*}$. Therefore, $f(x)=x$ because $V$ is regular. Theorem 2 is proved.

\section{Acknowledgement}

The authors are grateful to S. N. Sergeev for his kind help, and to the anonimous referee for careful reading and useful remarks on the previous version of the paper.

\section{References}

[1] J. Schauder, Der Fixpunktsatz in Funktionalräumen, Studia Math. 2 (1930), 171-180.

[2] G.L. Litvinov and V.P. Maslov, Correspondence Principle for Idempotent Calculus and Some Computer Applications, (IHES/M/95/33), Institut des Hautes Etudes Scientifiques, Bure-sur-Yvettes, 1995. The same in: J. Gunawardena (Ed.), Idempotency, Publ. of the I. Newton Institute, Vol. 11, Cambridge University Press, Cambridge, 1998, p. 420-443. E-print arXiv:math.GMA/0101021.

[3] G.L. Litvinov and V.P. Maslov, Idempotent mathematics: the correspondence principle and its applications to computing, Russian Math. Surveys 51 (1996), 1210-1211.

[4] G.L. Litvinov, Maslov dequantization, idempotent and tropical mathematics: a brief introduction, Journal of Mathematical Sciences 140 (2007), no. 3, 426444. E-print arXiv:math.GM/0507014.

[5] V.N. Kolokoltsov and V.P. Maslov, Idempotent Analysis and Its Applications, Kluwer Acad. Publ., 1997.

[6] S.Y. Yakovenko and L.A. Kontorer, Nonlinear semigroups and infinite horizon optimization, in [7], p. 167-210. 
[7] V.P. Maslov and S.N. Samborskiı̌, Eds., Idempotent Analysis, Advances in Soviet Mathematics, Vol. 13, Amer. Math. Soc., Providence, RI, 1992.

[8] G.L. Litvinov, V.P. Maslov, and G.B. Shpiz, Linear functionals on idempotent spaces: An algebraic approach, Russian Acad. Sci. Dokl. Math. 58 no. 3 (1998), 389-391. E-print arXiv:math.FA/0012268.

[9] G.L. Litvinov, V.P. Maslov, and G.B. Shpiz, Tensor products of idempotent semimodules: An algebraic approach, Math. Notes 65, no. 3-4 (1999), 479489. E-print arXiv:math.FA/0101153.

[10] G.L. Litvinov, V.P. Maslov, and G.B. Shpiz, Idempotent functional analysis: An algebraic approach, Math. Notes 69, no. 5-6 (2001), 696-729. E-print arXiv:math.FA/0009128.

[11] L. Fuchs, Partially Ordered Algebraic Systems, Pergamon Press, Oxford et. al., 1963.

[12] S.N. Samborski1 and G.B. Shpiz, Convex sets in the semimodule of bounded functions, in [7], p. 135-137.

[13] G. Litvinov and V. Maslov (eds.), Idempotent Mathematics and Mathematical Physics, Contemp. Math. Vol. 377, Amer. Math. Soc., Providence, RI, 2005.

[14] G. Cohen, S. Gaubert, J.-P. Quadrat, and I. Singer, Max-plus convex sets and functions, in [13], p. 105-129.

[15] I. Singer, Abstract Convex Analysis. Wiley, 1997. 